Food allergy tests

\section{Challenging times for food allergy tests}

S Roberts

\section{A discussion of their risks and benefits}

F ood allergies represent a significant problem in the western world, affecting about $7 \%$ of children under 3 years of age. ${ }^{2}$ The impact of food allergies extend beyond the affected individual to their immediate family, as a great deal of anxiety is generated by the fear of a possible fatal food allergy and the practical problem of food avoidance. ${ }^{3-5}$ Consequently it is essential that a correct diagnosis be made.

\section{INTERPRETATION OF THE CLINICAL HISTORY}

Diagnosing immediate hypersensitivity to food should not be expected to cause difficulty with the onset of well characterised symptoms occurring only minutes after ingestion. The observation that a positive diagnosis of food allergy could be confirmed in less than $50 \%$ of cases diagnosed by clinical history illustrates that the true picture is more complex and that diagnostic testing is required to confirm or refute the presence of allergy. ${ }^{67}$

There are several reasons for getting the diagnosis wrong. Foods are rarely eaten singly, and unravelling the allergen in a meal requires detailed knowledge of food manufacture as well as food recipes. Cooking a food changes the protein structure and this may affect the allergenic properties, so that, for example pasteurised milk becomes less likely to provoke an allergic reaction than unheated milk, but roasted peanuts are more likely to cause symptoms than untreated ones. ${ }^{8}$

Interpretation of allergic symptoms frequently causes difficulty. Eczematous patients, many of whom suffer from food intolerance, experience perioral urticaria and contact irritation provoked by fruit juices and other irritants. Patients with the oral allergy syndrome describe intra-oral pruritus and pharyngeal swelling which is a localised mucosal response without systemic involvement and related to the presence of type I IgE mediated hypersensitivity. ${ }^{9-11}$ Patients with ordinary or physical urticaria not infrequently attribute their rash to a food allergy, though an allergic cause can be demonstrated in less than $25 \%$. Symptoms of anaphylaxis may also be confused with generalised urticaria, acute anxiety, vasovagal attacks, mastocytosis, or hereditary angioedema. ${ }^{12}$

A further complication in the interpretation of food allergy symptoms in children is the dynamic nature of the allergic response which changes with time; the acquisition of food tolerance and amelioration of symptoms is reported in children with all types of food allergy. ${ }^{13} 14$

\section{EVALUATING FOOD ALLERGY TESTS}

Although the clinical history retains a fundamental place in diagnosis, we should not become complacent where there is an equal chance of the diagnosis being right or wrong. Are our patients served any better by diagnostic tests? None of the routinely available tests using allergen extract pricked into the dermis or measurement of specific serum IgE antibody in serum discriminate between sensitisation and clinical reactivity. False negative tests other than those attributable to poor technique or laboratory error are comparatively rare, but important because of the inherent risk of a severe allergic reaction in a patient who has been mistakenly reassured. ${ }^{15}$

False positive tests are numerically much more of a problem. The probability of finding a positive allergy test increases in patients with eczema and in patients with higher serum levels of IgE immunoglobulin, but the specificity of these tests varies between $49 \%$ and $95 \%$, depending on the size of the skin test wheal diameter and proportion of specific IgE antibody, and patient selection. $^{6716}$

In studies examining the relation between values obtained by allergy testing with the response to oral food challenge (OFC), the likelihood of a positive challenge can be predicted in up to $95 \%$ of selected patients by careful interpretation of results, but the studies are not consistent and vary with the prevalence of allergy in the population. ${ }^{16-18}$ A prospective study of OFC in patients who were predicted to react according to specific IgE levels found good positive prediction of symptomatic allergy but low sensitivity, so antibody tests were not useful in excluding a food allergy. Furthermore there was no association between specific IgE concentration and the severity of symptoms triggered by the OFC. Recent data from one centre measuring food specific IgE values has established predictive thresholds for allergic response which has enabled a $50 \%$ reduction in the need for food challenges. ${ }^{19}$ This seemingly important advance has not been universally applicable to different study populations. A reviewer of more recently published studies has suggested that each allergy centre should establish their own data for allergic thresholds and that food challenges will continue to be standard practice. ${ }^{19 a}$

In practice, sensitisation as defined by positive skin prick test and specific IgE antibody is encountered in many more asymptomatic individuals than in patients who show symptoms so that interpreting results is unreliable without a convincing clinical history. This creates the possibility of a false positive test supporting a mistaken diagnosis based on clinical symptoms encountered in up to $30 \%$ of cases. ${ }^{20}$

The skin prick testing procedure is widely applied and poses an extremely small risk to patients though it may rarely provoke a generalised allergic reaction in young infants. ${ }^{21}$

The real problem is that neither the size of the skin test reaction, nor the concentration of $\operatorname{IgE}$ antibody will answer the questions which patients most frequently ask in the allergy clinic. "Does my child have a food allergy, what will happen to my child if they eat the food, and how much or little food is necessary to produce a reaction?", cannot be answered without recourse to oral food challenge (OFC).

\section{USEFULNESS OF FOOD CHALLENGE TESTS}

A discussion of the relative merits of open and double blind placebo controlled challenge procedures is described elsewhere. The importance of the latter as a research tool and gold standard of food allergy diagnosis is established, though there are dose limitations and false negative tests are described. ${ }^{7}{ }^{19}$ By implication, research ethical committees accept that researchers will have obtained legally valid consent for OFC and that the procedure satisfies established ethical criteria; that the information obtained by the test is useful and that it cannot be obtained in any other way, and that children are exposed to the lowest risk that is consistent with obtaining the data. ${ }^{22}{ }^{23}$

In the clinical situation, open food challenge is sufficient in most of the patients with suspected allergy to 
confirm a diagnosis and to answer the most frequently asked questions.

Response to OFC may be absent, minimal, or severe as defined by need for emergency treatment of anaphylaxis as outlined in previous reports. ${ }^{24}$ The response may be more or less severe than predicted from previous history as illustrated in children with peanut allergy. Forty four per cent of those with a mild index reaction had a more severe reaction on follow up, ${ }^{25}$ though other studies have shown that up to $20 \%$ of patients with previous history show spontaneous resolution. ${ }^{1326}$

There is such a variable natural history for food allergies in children that OFC is often the most accurate and informative method of providing useful and contemporaneous advice to parents. ${ }^{27}$ Of the known limitations to the OFC procedure, an inadequate amount of food ingested is most likely to restrict usefulness of the test. Replication of clinical reactions requires up to $8 \mathrm{~g}$ of dried food or $60 \mathrm{~g}$ of wet food. $^{7}$ These amounts are difficult to achieve in practice because patients are reluctant to ingest foods which they find distasteful or uncomfortable, even in the absence of an allergic reaction. Anxiety symptoms are easily confused with allergic symptoms, so that even experienced clinicians may have difficulty in interpretation of events unless they are able to directly observe the patient throughout the entire procedure. Delayed reactions occurring several hours after ingestion are another source of error in children with cows' milk allergy, and patients must be informed of the potential for late symptoms. ${ }^{28}$ Despite these restrictions, false negative results are unusual, ${ }^{72}$ as are reports of reactions to placebo. ${ }^{19}$

The accepted indications for OFC are to confirm symptomatic food allergy or to demonstrate resolution of allergy, and a single test with sufficient food is adequate for this purpose. Validation of OFC by repeated challenge to show reproducible results is impractical and usually unethical. It is therefore not known if the test can be used reliably to demonstrate thresholds for the amount of food required to provoke symptoms, ${ }^{30}$ and unexpectedly severe reactions occurred when patients were rechallenged with food mixed with a high fat content. ${ }^{31}$ The authors suggest that early warning signals such as oral irritation are masked when lipophilic food allergens are consumed in a fatty meal. In these patients the OFC may yield useful information by demonstrating if the reaction occurs to traces of the food (very sensitive) or only to much larger amounts.
OFC in children probably does help to show if severity of allergy is declining with age, but symptoms on accidental ingestion may be worse than expected, for example during asthma exacerbation or after exercise. It is unwise to predict severity of future reactions from the outcome of challenge testing alone, and anaphylaxis treatment plans should be based on clinical assessment of risk ${ }^{32}{ }^{33}$ and knowledge of the natural history of the specific provoking allergen.

There are no published studies of patients' quality of life assessments following OFC, but our own unpublished data show that families value objective and contemporaneous information. In a recent survey of families of allergic children in South Manchester, 41 questionnaires were received from 83 patients, of which $85 \%$ reported that information from the challenge test was useful. Within the whole group 53\% had no reaction to food, but $24 \%$ had reactions needing urgent treatment. For these families their main concerns were uncertainty and lack of information, rather than a fear of the challenge procedure. Our low response rate introduces possible observer bias, and further evidence for the effectiveness of OFC would be useful.

\section{RISKS AND ETHICS OF FOOD CHALLENGES}

The allied issues of clinical risk assessment and informed consent for OFC are perhaps the most important and controversial ones confronting the paediatric allergist today. A consensus of clinical practice and recommendation is urgently required, but is unlikely to emerge until clinical allergy achieves specialty status in the Royal Colleges.

That severe allergic reactions occur during OFC is not in doubt, ${ }^{17}{ }^{35}$ and the procedure must take place in appropriately staffed children's units with adequate resuscitation facilities. Such reactions appear to be treated effectively without harmful effects to the patient, with no cases of very severe or fatal allergic reactions identified in a two year period in the UK and Ireland. ${ }^{24}$ Is this apparently reassuring finding a reflection of our reluctance to investigate children when the tests may put them at risk?

Most clinicians would hesitate to challenge children with a recent history of severe reaction and those with severe or unstable asthma. ${ }^{20}$ However, a number of other risk factors may also predispose a child to greater risk of anaphylaxis during OFC, such as a history of increasingly severe reactions, exquisite sensitivity to food, medication with $\beta$ adrenergic antagonists, and after elimination diets. ${ }^{34}$ There are very few published audits of OFC; the available data suggest that there is a $1 \%$ risk of severe reaction requiring treatment with adrenaline. ${ }^{35}$

Analysis of food challenge outcome in 59 consecutive patients in South Manchester showed a low incidence of severe reactions defined as needing emergency treatment with adrenaline or bronchodilator $(10.2 \%)$ and a high incidence of non-reactors (50.9\%), consistent with previous reports. ${ }^{7}$ In our experience, children with putative risk factors such as well controlled asthma, previous history of severe reaction in early childhood, or strongly positive skin prick test, did not have a different outcome to those children with no apparent risk factors, some of whom also had severe responses to challenge. Therefore, OFC can place any child at risk of an adverse event and we cannot confidently predict outcome, which is the principal reason for needing the information yielded by the test.

Opinion varies on selection of patients suitable for OFC (personal communications). A cautious approach will have the effect of overdiagnosing food allergy and the consequent need for dietary elimination. Such an approach satisfies the clinician's desire for prudence and may be acceptable to some families, though others will require a more definite diagnosis. We should question if the evidence justifies withholding OFC when parents and children would like to be tested and we can undertake the test in a far safer environment than in the home or school.

In addition, multiple food allergies can impose severe nutritional problems. Extensive elimination of foods based on false positive blood and skin tests cannot be justified, and it is unethical not to challenge these patients.

How should we advise patients when seeking informed consent for the procedure without introducing bias and personal prejudice? Our recommendations should be guided by the ethical principles of informing on what is known about the benefits and risks, and to do so in a way which preserves choice by informing appropriately. If we were to say to parents that there is a remote but actual risk of severe harm or death from the challenge, there would probably be few if any volunteers and these would be so anxious that the results might be uninterpretable. The more appropriate information is that the risk of severe or actual harm is extremely rare and unmeasurable, and that our experience of the tests is that they are safe.

The numbers of children experiencing potentially serious reactions during OFC in published reports ${ }^{2}$ and in our own 
patients is similar to the incidence of adverse drug reactions in hospitalised children. $^{36}{ }^{37}$ Unlike OFC, disasters and fatalities are reported with drug prescribing and in sufficient numbers to be a leading cause of death, though parents are not often aware of these statistics when we prescribe for their children. ${ }^{38} 39$

Many investigative procedures in children, such as endoscopy under general anaesthesia or injection of drugs in radiological contrast media also carry a small risk, the available evidence being that food challenge tests are actually safer than any of these. ${ }^{38} 40$

Few medical interventions are entirely free of risk and our role should be to enable patients to understand what we know about the balance of risk and benefit. ${ }^{41}$ Parents will naturally seek to protect their children from risk. In the context of food allergy, knowledge of the real risk of anaphylaxis can be more important to them than our reservations about the hazards of the only test which can at present help to provide them with the information they need.

Arch Dis Child 2005;90:564-566.

doi: 10.1136/adc.2003.036814

Correspondence to: Dr S Roberts, Department of Paediatrics, Wythenshawe Hospital, Southmoor Road, Manchester M23 9LT, UK; stephen.roberts@smuht.nwest.nhs.uk

Competing interests: none declared

\section{REFERENCES}

1 Sampson HA. Food allergy. Part 1. Immunopathogenesis and clinical disorder. J Allergy Clin Immunol 1999; 103:717-28.

2 Sampson HA. Use of food allergy tests in children. Lancet 2001;358:1832-3.

3 Hourihane JO'B. Community management of severe allergies must be integrated and comprehensive and must consist of more than epinephrine. Allergy 2001;56:1023-5.

4 Hourihane JO'B, Kilburn SA, Dean P, et al. Clinical characteristics of peanut allergy. Clin Exp Allergy 1997;27:634-9.

5 Sampson HA, Mendelson L, Rosen JP. Fatal and near-fatal anaphylactic reactions to food in children and adolescents. N Engl J Med 1992;327:380-4.

6 Sampson HA. Food allergy. Part 2. Diagnosis and management. J Allergy Clin Immunol 1999; 103:981-99.

7 Allan Bock SA, Atkins FM. Patterns of food hypersensitivity during 16 years of double blind placebo controlled food challenges. J Pediatr 1990;117:561-7.

8 Hefle SL. Impact of processing on food challenge. Adv Exp Med Biol 1999;459:107-19.

9 Dreborg S, Foucard T. Allergy to apple, carrot and potato in patients with pollen allergy. Allergy 1982;37:437-43.

10 Powe DG, Jagger $C$, et al. Localised mucosal allergic disease in the absence of systemic responses for atopy. Clin Exp Allergy 2003:33:1374-9.

11 Anhoej C, Backer V, Nolte H. Diagnostic evaluation of grass and birch allergic patients with oral allergy syndrome. Allergy 2001;56:548-52.

12 Walter S, Sheik A. Managing anaphylaxis. Clin Exp Allergy 2003;33:1015-18.

13 Skolnick H, Barnes-Koerner C, ConnoverWalker MK, et al. The natural history of peanut allergy. J Allergy Clin Immunol 2000; 105(suppl): 189.

14 Host A, Halken S, Jacobsen HP, et al. The natural course of cow's milk protein allergy. J Allergy Clin Immunol 1997:99(suppl):490.

15 Hill DJ, Duke AM, Hosking CS, et al. Clinical manifestations of cow's milk allergy in childhood. II. Diagnostic value of skin test and RAST. Clin Allergy 1988;18:481-90.

16 Sporick R, Hill DJ, Hosking CS. Specificity of allergen skin testing in predicting positive open challenges to egg, milk and peanut in children. Clin Exp Allergy 2000;11:1495-8.

17 Sampson HA, Ho DJ. Relationships between food specific lgE concentrations and the risk of positive food challenges in children and adolescents. J Allergy Clin Immunol 1997;100:444-51.

18 Rance F, Abbal M, Lauwers-Cancès V. Improved screening for peanut allergy by the combined use of skin prick test and specific lgE assays. J Allergy Clin Immunol 2002; 109:1027-33.

19 Sampson HA. Utility of food-specific lgE concentrations in predicting symptomatic food allergy. J Allergy Clin Immunol 2001;107:891-6.

19a Eigenmann PA. Are specific immunoglobulin E titres reliable for the prediction of food allergy? Clin Exp Allergy 2005;35:247-9.

20 Sampson HA, Albergo R. Comparison of results of skin tests RAST and DBPCFC in children with atopic dermatitis. J Allergy Clin Immunol 1984;74:26-33.

21 Devenney I, Faith-Magnussen K. Skin prick test may give generalised allergic reactions in infants. Ann Allergy Asthma Immunol 2000;85:457-60.

22 Royal College of Physicians Ethics Advisory Committee. Guidelines for the ethical conduct of medical research involving children. Arch Dis Child 2002;82:177-82.

23 Everard ML. Ethical aspects of using radio labelling in aerosol research. Arch Dis Child 2003;88:659-66.

24 McDougall CF, Cant AJ, Colver AF. How dangerous is food allergy in childhood. Arch Dis Child 2002:86:236-9.

25 Vanderleek TK, Liu AH, Stefanski K, et al. The natural history of peanut allergy in young children and its association with serum peanut specific lgE. J Pediatr 2000;137:749-55

26 Hourihane JO'B, Roberts SA, Warner JO Resolution of peanut allergy. Case controlled study. BMJ 1998;316:1271-5.

27 Du Toit G, Lack G. Optimising the diagnosis of peanut and tree nut allergy. Clin Exp Immunol 2003:33:1019-22.

28 Hill I, Hosking CS. Some limitations of double blind placebo controlled food challenges in young children. J Allergy Clin Immuno 1991:87:136-7.

29 Caffarelli C, Petroccione T. False negative food challenges in children with suspected food allergy. Lancet 2001;358:1871-2.

30 Taylor SL, Hefle SL, Bindslev-Jensen, et al. Factors affecting the determination of threshold doses for allergenic foods. J Allergy Clin Immunol 2002; 109:24-30.

31 Grimshaw KEC, King RM, Nordlee JA, et al. Presentation of allergen in different food preparations affects the nature of the allergic reaction. Clin Exp Allergy 2003;33:1581-5.

32 Mclean-Tooke APC, Bethune CA, Fay AC, et al. Adrenaline in the treatment of anaphylaxis: what is the evidence? BMJ 2003;327:1332-5.

33 Pumphrey RS. Lessons for management of anaphylaxis from a study of fatal reactions. Clin Exp Allergy 2000;30:1 144-50.

34 Patel L, Raddivan FS, David TJ. Management of anaphylactic reactions to food. Arch Dis Child 1994;71:370-5.

35 Torr T, Gaughan M, Roberts G, et al. Food challenges: a review and audit. Paediatr Nurs 2002; 14:30-4.

36 Choonara I, Clarkson A, Provasi D, et al. Incidence of adverse drug reactions in paediatric in/out-patients. Br J Clin Pharmacol 2001;52:77-83.

37 Kaushal R, Bates DW, Landrigan C, et at. Medication errors and adverse drug events in paediatric inpatients. JAMA 2001;285:2114-20

38 Cote CJ, Alderfer RJ, Notterman DA, et al. Sedation disasters: adverse drug reports in paediatrics. Anaesthesiology 1995;83:A1 183.

39 Lazarou J, Pomeranz BH, Corey PN. Incidence of adverse drug reactions in hospitalised patients. JAMA 1998;279:1200-5.

40 Cochrane Miller J. Minimising adverse reactions to contrast agents. Radiology Rounds 2003; 1:1-3.

41 Thornton H. Patients' understanding of risk. BMJ 2003;327:693-4. 